\title{
ON AN ERROR BY ANGUS E. TAYLOR
}

\author{
Pádraig Kirwan
}

\section{Introduction}

Mathematics is a difficult subject to study in any great depth. It is undoubtedly the subject that causes most anxiety to second and third level students. In the U.S.A. 'Math Anxiety' is frequently spoken about in the same way as appendicitis or the measles. It is generally not terminal but it is liable to strike almost anywhere. Much of this problem lies in the nature of mathematics. The answer to a mathematical question is rarely subjective, usually one is either right or wrong. A well formed question should elicit a precise and logical answer. Consequently there are two aspects to the mathematical development of an individual; the first is the ability to construct well formed questions and the second is to answer such questions in a consistent and clear manner. The failure of most people to master the first of these skills is the main reason why mathematics classes usually consist of one person, the lecturer, trying to impart mathematical knowledge to an audience, the students, in a passive manner. The reason for this is that most students are afraid to ask 'silly' questions. Thankfully I have never been shy of asking questions that were less than sensible and more often than not I have learned from the experience.

When an English or history teacher asks a question it frequently provokes a discussion, when a mathematics teacher asks a question it invariably silences the room. A fear descends on most students. This is usually a fear of getting the answer wrong.

1991 Mathematics Subject Classification. Primary 46G20. Secondary 46B28.

Key words and phrases. Complexification, normed spaces. 
Mathematics is black and white. In a discussion one can have a dissenting opinion, in mathematics one can be wrong. This fear can stifle the mathematical progression of many students at all levels.

A number of years ago I heard the following remark: 'When a mathematics student finishes their bachelor's degree they think they know everything. When a mathematics student finishes their master's degree they think they know nothing and when a mathematics student finishes their Ph.D. they realize that nobody else knows anything either'. This probably overstates the situation but it is not completely devoid of merit (discuss!). I am frequently asked: 'How does one do research in mathematics? Hasn't everything of a mathematical nature been discovered?'These were questions I asked myself when I started my master's degree. When I finished that course of study I was amazed at how the allconsuming bachelor's degree had barely scratched the surface of the subject. When I started my doctorate the fear I mentioned earlier struck me for the first time. I had become afraid that all my questions would be silly and that all my answers would be wrong. Many of my questions were silly and a lot of my answers were wrong, however by the end of my Ph.D. I realized that I was in good company in these respects.

Mistakes are not the domain solely of research students. It happens from time to time that experienced mathematicians also make mistakes and that these errors are not detected prior to publication. Such a mistake was made by Angus E. Taylor in the late 1930's. I will now examine this mistake and its correction in some detail.

\section{An easy mistake to make.}

The central theme of my thesis was the complexification of various mathematical objects. The word complexification was enough to intimidate most of my friends and non-mathematically minded colleagues. Consequently I don't think any of them have read the thesis from cover to cover, yet. Complexification essentially means associating a complex object such as a vector space, a norm, a linear operator, or a polynomial, with a real one in a system- 
atic manner. The complexification of a real vector space $E$ was straightforward to define and could be given by $E_{\mathbf{C}}=E \oplus i E$. If $z \in E_{\mathbf{C}}$ then there exists $x, y \in E$ such that $z=x+i y$. Multiplication by complex scalars was defined to be

$$
(a+i b)(x+i y)=a x-b y+i[a y-b x] \text { where } a, b \in \mathbf{R} .
$$

Initially I defined the complex norm on the complexification of the real normed space $\left(E,\|\|_{E}\right)$ to be

$$
\|z\|_{T}=\sqrt{\|x\|_{E}^{2}+\|y\|_{E}^{2}}
$$

This was the obvious natural extension of the modulus on complex numbers. With this norm I was able to show that the complexification of a linear operator had norm equal to that of the real linear operator and obtain estimates for the norms of complexified multilinear forms and polynomials. It was not long before inconsistent results were obtained and the fundamentals of the complexification process had to be re-examined. It soon became clear that the \|\|$_{T}$ norm did not respect complex scalar multiplication. I had carelessly only checked that

$$
\|i z\|_{T}=\|-i z\|_{T}=\|z\|_{T},
$$

which is clearly true but in hindsight it was not enough to show that \|\|$_{T}$ was not a complex norm.

Angus E. Taylor introduced this norm in 1937. He used complexifications to prove a generalized version of the CauchyRiemann equations.

Proposition 1. [8] Let $E$ and $F$ be real normed spaces and $D$ be a domain of $E_{\mathbf{C}}$. If $f: D \rightarrow F_{\mathbf{C}}$ is a function such that

$$
f(x+i y)=f_{1}(x, y)+i f_{2}(x, y)
$$

for $f_{1}, f_{2}: E \times E \rightarrow F$, then $f$ is analytic in $D$ if and only if $f_{1}$ and $f_{2}$ are continuous and have continuous first partial 'Gateaux' differentials at all points of $D$ and

$$
\begin{aligned}
\delta_{x} f_{1}(x, y ; \xi) & =\delta_{y} f_{2}(x, y ; \xi) \\
\delta_{y} f_{1}(x, y ; \xi) & =-\delta_{x} f_{2}(x, y ; \xi)
\end{aligned}
$$

holds in $D$ for all $\xi \in E$. 
If $E, F=\mathbf{R}$ then the Gateaux differentials reduce to the usual differentials one encounters in an advanced calculus class. Taylor's result is not valid because he uses \|\|$_{T}$ as a complex norm on $E_{\mathbf{C}}$ and $F_{\mathbf{C}}$. Indeed Taylor needs more than just a complex norm on the complexified spaces. He requires a complex norm \|\|$_{\mathbf{C}}$ on $E_{\mathbf{C}}$ such that

$$
\begin{array}{ll}
\text { (i) } & \|x\|_{\mathbf{C}}=\|x\|_{E} \forall x \in E \\
\text { (ii) } & \|x+i y\|_{\mathbf{C}} \geq\|x\|_{E},\|y\|_{E} \forall x, y \in E .
\end{array}
$$

In my thesis [2], I describe such a norm as desirable.

The following year Taylor made use of the \|\|$_{T}$ norm to obtain an estimate for the norm of a polynomial. We say that $P$ is an $n$-homogeneous polynomial from $E$ to $F$ if there exists a symmetric $n$-linear mapping $A: E^{n} \rightarrow F$ such that

$$
P(x)=A(x, \ldots, x) .
$$

We denote the space of $n$-homogeneous polynomials from $E$ to $F$ by $\mathcal{P}\left({ }^{n} E ; F\right)$. If $F$ is the scalar field, real or complex, then we denote this space by $\mathcal{P}\left({ }^{n} E\right)$. The norm of an $n$-homogeneous polynomial is usually given by $\|P\|=\sup _{\|x\|_{E} \leq 1}\|P(x)\|_{F}$. Using elementary calculus Taylor proves the following:

Proposition 2 [8] Let $P$ be an $n$-homogeneous polynomial on $E$. If $E_{\mathbf{C}}$ is endowed with the \|\|$_{T}-$ norm, then

$$
\left\|P_{\mathbf{C}}\right\| \leq 2^{\frac{n-1}{2}}\|P\|
$$

Once again this result is wrong because \|\|$_{T}$ is not a complex norm. However if we replace Taylor's norm with a desirable norm \|\|$_{\mathbf{C}}$ on $E_{\mathbf{C}}$ such that

$$
\|z\|_{\mathbf{C}} \geq\|z\|_{T} \forall z \in E_{\mathbf{C}}
$$

then Taylor's inequality is valid. Furthermore if we use a norm \|\|$_{\mathbf{C}}$ on $E_{\mathbf{C}}$ such that

$$
\|x+i y\|_{\mathbf{C}} \geq\left(\|x\|_{E}^{p}+\|y\|_{E}^{p}\right)^{\frac{1}{p}}
$$


then

$$
\left\|P_{\mathbf{C}}\right\| \leq 2^{\frac{n-1}{q}}\|P\|
$$

where $\frac{1}{p}+\frac{1}{q}=1$. In [2], I show that if we choose any desirable norm, then Taylor's constant should be replaced by $2^{n}$. This is done by first showing that

$$
P_{\mathbf{C}}(x+i y)=\frac{2^{n}}{2 \pi} \int_{0}^{2 \pi} P(x \cos \theta+y \sin \theta) e^{i n \theta} d \theta
$$

and then using the triangle inequality. Subsequently Gustavo Munoz [6] has found the best constant to be $2^{n-1}$ and that this constant is sharp.

Taylor's mentor, A. D. Michal, was the first to point out in 1940 in a paper with Davis and Wyman that the Taylor norm was deficient. In fact they tell us more.

Proposition $3[4]\left(E_{\mathbf{C}},\|\|_{T}\right)$ is a complex normed vector space if and only if $\left(E,\|\|_{E}\right)$ is an inner product space.

The subsequent year Michal and Wyman [5] used the following norm on the complexified normed space:

$$
\|z\|_{M W}=\sup _{\phi \in B_{E^{*}}} \sqrt{|\phi(x)|^{2}+|\phi(y)|^{2}} \text { where } z=x+i y \in E_{\mathbf{C}} .
$$

Michal and Wyman credit Taylor with this norm. This norm has been seen in many different guises over the past half century. Lindenstrauss and Tzafriri [3] describe the natural norm on the complexification of a lattice to be

$$
\|z\|_{L T}=\sup _{\theta}\|x \cos \theta+y \sin \theta\|_{E} \text { where } z=x+i y \in E .
$$

This is easily seen to be identical to the Michal and Wyman norm on the complexification of any real normed space. An alternative approach is to define a class of norms as follows:

$$
\begin{aligned}
& \|z\|_{p, q}=\left(\frac{1}{2 \pi} \int_{0}^{2 \pi}\left(\|x \cos \theta-y \sin \theta\|_{E}^{p}+\|x \sin \theta+y \cos \theta\|_{E}^{p}\right)^{\frac{q}{p}} d \theta\right)^{\frac{1}{q}} \\
& \|z\|_{p, \infty}=\sup _{\theta}\left(\|x \cos \theta-y \sin \theta\|_{E}^{p}+\|x \sin \theta+y \cos \theta\|_{E}^{p}\right)^{\frac{1}{p}}
\end{aligned}
$$


For $p=\infty$ these reduce to the following

$$
\begin{aligned}
\|z\|_{\infty, q} & =\left(\frac{1}{2 \pi} \int_{0}^{2 \pi} F(\theta) d \theta\right)^{\frac{1}{q}} \\
\|z\|_{\infty, \infty} & =\sup _{\theta}\|x \cos \theta-y \sin \theta\|_{E},
\end{aligned}
$$

where

$$
F(\theta)=\max \left\{\|x \cos \theta-y \sin \theta\|_{E},\|x \sin \theta+y \cos \theta\|_{E}\right\}^{q} .
$$

We denote the Lebesgue-Bochner space of $E$-valued $q$-integrable functions on the unit circle in the complex plane, $\mathbf{T}$ by $L_{q}(\mathbf{T}, E)$, where $\mathbf{T}$ is endowed with the normalized arc-length measure. Consequently if $z=x+i y \in E_{\mathbf{C}}$ we define $f_{z} \in L_{q}\left(\mathbf{T}, E \oplus_{p} E\right)$ by

$$
f_{z}\left(e^{i \theta}\right)=(x \cos \theta-y \sin \theta, x \sin \theta+y \cos \theta) .
$$

Then we get

Proposition $4[2]$ Let $\left(E,\|\|_{E}\right)$ be a real normed space. Then the mapping $z \in E_{\mathbf{C}} \rightarrow f_{z}$ defines an isometric embedding of $\left(E_{\mathbf{C}},\|\|_{p, q}\right)$ into $L_{q}\left(\mathbf{T}, E \oplus_{p} E\right)$.

Clearly this gives us another way of looking at the MichalWyman, Lindenstrauss-Tzafriri norm. I. E. Verbitskii gives the following representation of this norm in [9]:

$$
\|z\|_{v}=\sup _{\phi \in B_{E^{*}}}\left|\phi_{\mathbf{C}}(z)\right|
$$

where $\phi_{\mathbf{C}}$ is the complexification of $\phi$ defined by

$$
\phi_{\mathbf{C}}(x+i y)=\phi(x)+i \phi(y) .
$$

Verbitskii alludes to the fact that this is the smallest desirable norm on a complexified space. Therefore it is easy to see that it is identical to the Michal-Wyman norm. At the other end of the 
scale Verbitskii [9] gave the following representation of the largest desirable norm:

$$
\|z\|_{V}=\sup \{|\phi(z)|\},
$$

where $\phi$ is a complex linear mapping from $E_{\mathbf{C}}$ into $\mathbf{C}$ such that $\phi$ restricted to $E$ is a norm one real linear functional. The only other representation I have found of the largest desirable norm comes from the tensor product approach. The tensor product $E \otimes F$ is the space of linear functionals on $\mathcal{L}\left({ }^{2} E \times F\right)$ where

$$
x \otimes y(B)=B(x, y),
$$

for all $x \in E, y \in F, B \in \mathcal{L}\left({ }^{2} E \times F\right)$. A norm $\gamma$ on this space is called a reasonable crossnorm if it satisfies

$$
\gamma(x \otimes y) \leq\|x\|_{E}\|y\|_{F}
$$

and if $x^{*} \in E^{*}$ and $y^{*} \in F^{*}$, then $x^{*} \otimes y^{*} \in(E \otimes F, \gamma)^{*}$ and has functional norm less than or equal to $\left\|x^{*}\right\|_{E^{*}}\left\|y^{*}\right\|_{F^{*}}$. Since every desirable norm on $E_{\mathbf{C}}$ is a reasonable crossnorm on $E \otimes \ell_{2}^{2}$ we have that the projective tensor norm, $\pi$, is the largest desirable norm on $E_{\mathbf{C}}$. Similarly the injective tensor norm, $\varepsilon$, is the smallest and so coincides with the Michal-Wyman norm. A more complete picture is obtained if we look at the norms introduced by Lapresté and Saphar. These are defined as follows: for $1 \leq p, q \leq \infty$ with $\frac{1}{p}+\frac{1}{q} \geq 1$, take the unique $r \in[1, \infty]$ with

$$
\frac{1}{r}=\frac{1}{q}+\frac{1}{p}-1 \text { or equivalently } 1=\frac{1}{r}+\frac{1}{q^{\prime}}+\frac{1}{p^{\prime}}
$$

(where' denotes conjugacy) and define for normed spaces $E$ and $F$ and $z \in E \otimes F$

$$
\alpha_{p, q}(z)=\inf \left\{\ell_{r}^{n}\left(\lambda_{k}\right) w_{q^{\prime}}^{n}\left(x_{k}\right) w_{p^{\prime}}^{n}\left(y_{k}\right): z=\sum_{i=1}^{n} \lambda_{i} x_{i} \otimes y_{i}\right\}
$$

where

$$
\ell_{p}^{n}\left(x_{k}\right)=\left(\sum_{k=1}^{n}\left\|x_{k}\right\|_{E}^{p}\right)^{\frac{1}{p}}
$$

and 


$$
w_{p}^{n}\left(x_{k}\right)=\sup _{\phi \in E^{*}}\left(\sum_{k=1}^{n}\left|\phi\left(x_{k}\right)\right|^{p}\right)^{\frac{1}{p}}
$$

All these norms represent complex norms on $E_{\mathbf{C}}$. Clearly

$$
\alpha_{1,1}(z)=\pi(z)
$$

For more information on these norms we refer the reader to Defant and Floret [1]. Special cases of $\alpha_{p, q}$-norms are (for $1 \leq p \leq \infty$ )

$$
\begin{aligned}
& g_{p}(z)=\alpha_{p, 1}(z)=\inf \left\{\ell_{p}\left(x_{i}\right) w_{p^{\prime}}\left(t_{i}\right): z=\sum_{i=1}^{n} x_{i} \otimes t_{i}\right\} \\
& d_{p}(z)=\alpha_{1, p}(z)=\inf \left\{w_{p^{\prime}}\left(x_{i}\right) \ell_{p}\left(t_{i}\right): z=\sum_{i=1}^{n} x_{i} \otimes t_{i}\right\} \\
& w_{p}(z)=\alpha_{p, p^{\prime}}(z)=\inf \left\{w_{p}\left(x_{i}\right) w_{p^{\prime}}\left(t_{i}\right): z=\sum_{i=1}^{n} x_{i} \otimes t_{i}\right\}
\end{aligned}
$$

The manner in which these norms fit in is best seen by means of the following diagram.

Taylor's mistake, though obvious in hindsight, was not entirely without merit. If his norm had been complex then it would have been desirable and so many incorrect results could be corrected by replacing Taylor's norm with any of the myriad norms mentioned above. However, as recently as 1986, Guido Zapata [10] erred in a similar manner to Taylor. He defined the norm on the complexification of a strongly separating Banach Lattice algebra $A$ of real continuous functions on $E$ by

$$
\|f\|_{Z}=\|\operatorname{Re}(f)\|_{A}+\|\operatorname{Im}(f)\|_{A} .
$$

Zapata uses this to prove a generalization of the Stone-Weierstrass Theorem. His proof is obviously not valid but it is easily corrected. The reviewer's remark neatly sums up the situation: 'The author is in good company when it comes to putting a 'norm' on the 
complexification of a real Banach space (p.928), but this oversight probably does not affect the validity of his work in that direction'.

It is often the case that an incorrect solution to a scientific problem is just as interesting as a correct one. Quite often we learn more from the questions we get wrong than from those we get right. After all there is no point in making mistakes if we don't learn from them and since nobody will live long enough to make all possible mistakes it is important to learn from other people's mistakes also. As they say, 'To err is human'.

\section{References}

[1] A. Defant and K. Floret, Tensor Norms and Operator Ideals. NorthHolland Math. Studies (176). North-Holland: Amsterdam, 1993.

[2] P. Kirwan, Complexification of Multilinear and Polynomial Mappings on Normed Spaces. Ph.D. Thesis, N.U.I., Galway, 1997.

[3] J. Lindenstrauss and L. Tzafriri, Classical Banach Spaces, I. SpringerVerlag: Berlin-Heidelberg-New York, 1977.

[4] A. D. Michal, R. Davis and M. Wyman, Polygenic functions in general analysis, Ann. Scuola Norm. Sup. Cl. Sci 9 (1940), 97-108.

[5] A. D. Michal and M. Wyman, Characterization of complex couple spaces, Ann. of Math. 42 (1941), 247-250.

[6] G. Munoz, Y. Sarantopolous and A. Tonge, Complexifications of real Banach spaces, polynomials and multilinear maps, preprint, 1998.

[7] A. E. Taylor, Analytic functions in general analysis, Ann. Scuola Norm. Sup. Cl. Sci 6 (1937), 277-292.

[8] A. E. Taylor, Additions to the theory of polynomials in normed linear spaces, Tôhoku Math. J. 44 (1938), 302-318.

[9] I. E. Verbitskii, Some relations between the norm of an operator and that of its complex extension, Mat. Issled Vyp. 42 (1976), 3-12.

[10] G. Zapata, The Stone-Weierstrass theorem and a class of Banach lattice algebras, in Aspects of Math. \& Appl., 913 -942. North-Holland Math. Library (34). North-Holland: Amsterdam, 1986. 
Pádraig Kirwan

Department of Physical and Quantitative Sciences

Waterford Institute of Technology

Cork Road

Waterford

Ireland 